\title{
Retraction Note: Diagnostic accuracy of 320-row computed tomography as compared with invasive coronary angiography in unselected, consecutive patients with suspected coronary artery disease
}

\author{
F. Pelliccia $\cdot$ V. Pasceri $\cdot$ A. Evangelista $\cdot$ A. Pergolini $\cdot$ F. Barillà $\cdot$ \\ N. Viceconte · G. Tanzilli • M. Schiariti · C. Greco • C. Gaudio
}

Published online: 27 March 2014

(C) Springer Science+Business Media Dordrecht 2014

Retraction to: Int J Cardiovasc Imaging (2013) 29:443-452

DOI 10.1007/s10554-012-0095-4

Diagnostic Accuracy of 320-row Computed Tomography as Compared with Invasive Coronary Arteriography in Unselected, Consecutive Patients with Suspected Coronary Artery Disease by F. Pelliccia, V. Pasceri, A. Evangelista, A. Pergolini, F. Barilla, N. Viceconte, G. Tanzilli, M. Schiariti, C. Greco, C. Gaudio published online in the International Journal of Cardiovascular Imaging on July 18, 2012, DOI 10.1007/s10554-012-0095-4 has been retracted at the request of the Editor-in-Chief as it contains large portions of text that have been duplicated from
Noninvasive Coronary Angiography by 320-Row Computed Tomography with Lower Radiation Exposure and Maintained Diagnostic Accuracy. Comparison of Results with Cardiac Catheterization in a Head-to-Head Pilot Investigation by M. Dewey, E. Zimmermann, F. Deissenrieder, M. Laule, H.-P. Dübel, P. Schlattmann, F. Knebel, W. Rutsch and B. Hamm published online in Circulation: Cardiovascular Imaging on August 24, 2009, DOI 10.1161/ CIRCULATIONAHA.109.859280.
The online version of the original article can be found under doi:10.1007/s10554-012-0095-4.

F. Pelliccia $(\bowtie) \cdot$ A. Evangelista · A. Pergolini · F. Barillà ·

N. Viceconte · G. Tanzilli - M. Schiariti - C. Greco - C. Gaudio Department of Heart and Great Vessels Attilio Reale, Sapienza University, Via Tommaso Inghirami 85, 00179 Rome, Italy

e-mail: f.pelliccia@mclink.it

V. Pasceri

Department of Cardiovascular Diseases,

San Filippo Neri Hospital, Rome, Italy
A. Evangelista $\cdot$ A. Pergolini
Department of Radiology, Villa Sandra Hospital, Rome, Italy
C. Greco $\cdot$ C. Gaudio
Eleonora Lorillard Spencer Cenci Foundation, Rome, Italy 